Schmerz 2020 $34: 373-375$

https://doi.org/10.1007/s00482-020-00505-6

๑) Springer Medizin Verlag GmbH, ein Teil von Springer Nature 2020

\title{
B. Zernikow
}

Deutsches Kinderschmerzzentrum und Kinderpalliativzentrum Datteln, Vestische Kinder- und Jugendklinik Datteln, Lehrstuhl für Kinderschmerztherapie und Pädiatrische Palliativmedizin, Universität Witten/Herdecke, Datteln, Deutschland

\section{Selten ist häufig! Schmerzen und Schmerzbehandlung bei seltenen Erkrankungen}

Schmerz ist insbesondere dann ein führendes leidvolles Krankheitssymptom bei seltenen Erkrankungen, wenn diese mit massiven Gewebsverletzungen und Entzündungen einhergehen. Prototypisch hierfür steht die Epidermolysis bullosa $(E B)$, eine seltene, hereditäre, blasenbildenden Erkrankung [9]. Schmerz entsteht durch neu auftretende Blasen - vor allem, wenn sie superinfizieren - und den täglichen oft stundenlangen Verbandswechsel. Martin et al. kommen in einem aktuellen Review über Epidermolysis bullosa zu der Aussage: „The strongest conclusion to be drawn from this review is that pain is a key theme for many people affected by EB", und sie fahren fort: „We recommend that pain management is a priority within clinical management and research aimed at improving the psychosocial well-being of people with EB" [9].

Bei den seltenen Schmerzerkrankungen Erythromelalgie, „paroxysmal extreme pain disorder", den seltenen primären Kopfschmerzerkrankungen wie Clusterkopfschmerz, paroxysmale $\mathrm{He}$ mikranie und SUNCT-Syndrom oder dem komplex regionalen Schmerzsyndrom (CRPS) sind der Schmerz, seine Charakteristik, sein zeitlicher Verlauf, mögliche Trigger und Begleitphänomene krankheitsdefinierend. Es ist gut, dass es in Deutschland Fachzentren für seltene Erkrankungen gibt, die sich der Patienten mit diesen oft äußerst schwer zu behandelnden seltenen Schmerzerkrankungen annehmen [1]. Der Gemeinsame Bundesausschuss hat hierfür am 5. De- zember 2019 die rechtliche Grundlage geschaffen [5].

Gar nicht so selten begegnen uns am Deutschen Kinderschmerzzentrum $\mathrm{Pa}$ tienten, die eine aktuell gut behandelte, wenig krankheitsaktive seltene Erkrankung haben, aber trotz fehlender Krankheitsaktivität eine hohe schmerzbedingte Funktionseinschränkung aufweisen - und zwar sowohl sozial als auch emotional. Diese Patienten erfüllen oft die Diagnosekriterien einer chronischen Schmerzstörung nach F45.41 [2]. Ausgangspunkt der chronischen Schmerzstörung sind die seltene Erkrankung und die damit einhergehenden psychischen Belastungen sowie chronische Schmerzerfahrungen. Gerade auch bei Patienten mit einer Hämophilie, wie sie im Beitrag von Emmert und Mitarbeitern beschrieben werden, haben Schmerzforscher wiederholt eine signifikant abgesenkte mechanische Schmerzschwelle nachgewiesen [6]. Solche peripheren und zentralen Schmerzsensibilisierungen sind auch für Kinder mit CRPS mittels funktionellem MRT gezeigt worden. Die Patienten wiesen trotz erfolgreicher Behandlung eine erhöhte zentrale neuronale Konnektivität sowie abgesenkte Schmerzschwellen auf [8]. Der Goldstandard der Schmerzbehandlung dieser Patienten ist die (teil)stationäre multimodale interdisziplinäre Schmerztherapie, wie sie von der Ad-hoc-Kommission der Deutschen Schmerzgesellschaft beschrieben wurde [3]. 


\section{Besondere Situationen: Kinder mit seltenen Erkrankungen und ihre Schmerztherapie}

Kinder mit seltenen Erkrankungen stellen eine besondere Herausforderung dar. Mehrere Projekte des Innovationsfonds widmen sich aktuelle dieser Problematik. Die Projekte:

1. „ZSE-DUO - Duale Lotsenstruktur zur Abklärung unklarer Diagnosen in Zentren für Seltene Erkrankungen",

2. „TRANSLATE-NAMSE - Verbesserung der Versorgung von Menschen mit seltenen Erkrankungen durch Umsetzung von im nationalen Aktionsplan (NAMSE) konsentierten Maßnahmen" sowie

3. „CARE-FAM-NET - Kinder mit seltenen Erkrankungen, deren Geschwister und Eltern"

haben das Ziel, die Versorgung dieser Patienten zu verbessern.

Schmerztherapie ist oft wichtiger Teil der dort definierten regulären Behandlung, wobei insbesondere die fehlende Erforschung und Zulassung von Analgetika ein Problem darstellt, welches auch Emmert und Kollegen in ihrem CMEBeitrag anreißen. Die Zulassung von CoAnalgetika und Analgetika im Kindesund Jugendalter muss nicht nur das Altersspektrum fokussieren, sondern auch die Grunderkrankung oder zu behandelnde Symptomatik. So kann ein Medikament für die antikonvulsive Behandlung im Kindesalter zugelassen sein, aber nicht für die Schmerztherapie, was bei der Aufklärung und Anwendung bedacht werden muss. Anders als viele Ärztinnen und Ärzte vermuten, ist Amitriptylin im Kindes- und Jugendalter für keine Indikation zugelassen.

Viele schwere seltene Erkrankungen münden schon früh in einer Zerstörung des zentralen Nervensystems mit dem klinischen Bild einer schwersten Mehrfachbehinderung. Eine verbale Kommunikation ist dann oft nicht mehr möglich. Die Schmerzeinschätzung erfolgt über Fremdbeobachtung, beispielsweise durch die standardisierte Fremdeinschätzung mittels Pediatric Pain Profile [7]. Bei diesen Patienten basiert die Behandlung des Symptoms
„Unruhe mit schmerztypischem Verhalten" leider nicht auf wissenschaftlichen Daten, sondern auf Erfahrungswissen der Beteiligten, sodass bei demselben klinischen Bild Experten zu vielen unterschiedlichen Empfehlungen kommen [10]. Insbesondere für diese extrem vulnerable Gruppe von Menschen mit seltenen Erkrankungen und schwerster Mehrfachbehinderung bedarf es konzertierter Forschungsanstrengungen.

Es ist der Verdienst der Autoren um D. Emmert (https://doi.org/10.1007/ s00482-020-00487-5), dass sie sich dem wichtigen Thema der Schmerztherapie bei seltenen Erkrankungen zugewandt haben.

\section{Korrespondenzadresse}

\section{Prof. Dr. B. Zernikow}

Deutsches Kinderschmerzzentrum und Kinderpalliativzentrum Datteln, Vestische Kinder- und Jugendklinik Datteln, Lehrstuhl für Kinderschmerztherapie und Pädiatrische Palliativmedizin, Universität Witten/Herdecke Dr.-Friedrich-Steiner-Str. 5, 45711 Datteln, Deutschland

B.Zernikow@kinderklinik-datteln.de

Interessenkonflikt. B. Zernikow gibt an, dass kein Interessenkonflikt besteht.

\section{Literatur}

1. Fachzentren für seltene Erkrankungen. https:// www.kinderklinik-datteln.de/leistungen/ fachzentren-fuer-seltene-erkrankungen/. Zugegriffen:27. Aug. 2020

2. ICD-10-GM-2020 F45.41 Chronische Schmerzstörung mit somatischen und psychischen Faktoren - ICD10. https://www.icd-code.de/icd/code/F45 41.html. Zugegriffen: 27. Aug. 2020

3. Arnold B, Brinkschmidt T, Casser H-R et al (2014) Multimodale Schmerztherapie für die Behandlung chronischer Schmerzsyndrome. Schmerz 28:459-472

4. Bösch A, Wager J, Zernikow B, Thalemann R, Frenzel H, Krude H, Reind T (2018) Life-limiting conditions at a University pediatric tertiary care center: a cross-sectional study. J Palliat Med 21:169-176

5. Bundesausschuss Gemeinsamer (2019) Beschluss des Gemeinsamen Bundesausschusses über die Erstfassung der Regelungen zur Konkretisierung der besonderen Aufgaben von Zentren und Schwerpunkten gemäß § 136c Absatz 5 SGB V (Zentrums-Regelungen)

6. Hilberg T, Czepa D, Freialdenhoven D, Boettger MK (2011) Joint pain in people with hemophilia depends on joint status. Pain 152:2029-2035

7. Hunt A, Wisbeach A, Seers K, Goldman A, Crichton N, Perry L, Mastroyannopoulou K (2007) Development of the aediatric pain profile: role of video analysis and saliva cortisol in validating a tool to assess pain in children with severe neurological disability. J Pain Symptom Manage 33:276-289

8. Linnman C, Becerra L, Lebel A, Berde C, Grant PE, Borsook D (2013) Transient and persistent pain induced connectivity alterations in pediatric complex regional pain syndrome. PLoS One 8:e57205

9. Martin K, Geuens S, Asche JK, Bodan R, Browne F, Downe A, Garcia Garcia N, Jaega G, Kennedy B, Mauritz PJ, Perez F, Soon K, Zmazek V, MayreChilton KM (2019) Psychosocial recommendations for the care of children and adults with epidermolysis bullosa and their family: evidence based guidelines. Orphanet J Rare Dis 14:133

10. Siden HB, Carleton BC, Oberlander TF (2013) Physician variability in treating pain and irritability of unknown origin in children with severe neurological impairment. Pain Res Manag 18:243-248 
Hier steht eine Anzeige.

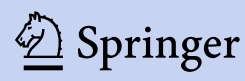

